\title{
Optimal Location and Sizing of Distributed Storage Systems in Active Distribution Networks
}

\author{
M. Nick, Student Member, IEEE, M. Hohmann, R. Cherkaoui, Senior Member, IEEE \\ and M. Paolone, Senior Member, IEEE \\ Distributed Electrical System Laboratory (DESL) \\ École Polytechnique Fédérale de Lausanne EPFL \\ Lausanne, Switzerland \\ \{mostafa.nick; marc.hohmann; rachid.cherkaoui; mario.paolone\}@epfl.ch
}

Nomenclature

DSS Distributed Storage System

$t \quad$ Index of time

$i, j \quad$ Index of nodes

$V_{i, t} \quad$ Voltage of bus $i$ at time $t$

$V_{\text {ref }} \quad$ Magnitude of the voltage reference

$W_{\text {vol }} \quad$ Weight-coefficient of voltage minimization

$W_{\text {loss }} \quad$ Weight-coefficient of loss minimization

$\Gamma_{\text {loss }} \quad$ Network losses

$W_{E P} \quad$ Weight-coefficient of energy-cost minimization

$F_{G A} \quad$ Value of fitness function

$C_{t}^{E} \quad$ Cost of energy from the grid at time $t$

$E_{t} \quad$ Energy flow towards an external grid

$\eta_{i} \quad$ DSS energy storage efficiency

$E_{D S S_{i}}^{0} \quad$ Initial energy stored in all DSSs connected to $i$ th bus

$E_{D S S_{i}}^{\max } \quad$ Maximum capacity of the DSS reservoir connected to $i$ th bus

$E_{D S S_{i}}^{\min } \quad$ Minimum capacity of the DSS reservoir connected to $i$ th bus

$E_{D S S_{i}}^{t} \quad$ Energy stored in the DSS connected to $i$ th bus at time $t$

$P_{D S S_{i}}^{\max } \quad$ Maximum active power capability of the DSS connected to $i$ th bus

$P_{D S S_{i}}^{\min } \quad$ Minimum active power capability of the DSS connected to $i$ th bus

$P_{D S S_{i}}^{t} \quad$ Active power produced/absorbed by the DSS connected to $i$ th bus at time $t$

$Q_{D S S_{i}}^{t} \quad$ Reactive power produced/absorbed by the DSS connected to $i$ th bus at time $t$
$Q_{L_{i, t}} \quad$ Reactive power absorbed at $i$ th bus at time $t$

$Q_{G_{i, t}} \quad$ Reactive power generated at $i$ th bus at time $t$

$P_{L_{i, t}} \quad$ Active power absorbed at $i$ th bus at time $t$

$P_{G_{i, t}} \quad$ Active power generated at $i$ th bus at time $t$

$\delta_{i, t} \quad$ Voltage phase of bus $i$ at time $t$

$G_{i, j} \quad$ Conductance of the line between buses $i$ and $j$

$B_{i, j} \quad$ Susceptance of the line between buses $i$ and $j$

$C_{D S S_{i}}^{\max } \quad$ Power capability limit of the DSS connected to $i$ th bus

Abstract - Energy balance and ancillary services provided by distributed storage systems to active distribution networks represent two aspects of a single problem that needs to be properly treated in view of the typical distribution networks parameters. In this context, the paper focuses on the problem of the optimal siting and sizing of distributed storage systems. In particular, the paper proposes the formulation of a problem that accounts: (i) the voltage support of storage systems to the grid, (ii) the network losses and (iii) the cost of the energy-flow towards the external grid. As the formulated problem is mixedinteger, non-convex and non-linear, its solution requires the adoption of heuristic techniques. In this respect, a two-stage iterative procedure is proposed. The first stage of the procedure utilizes a genetic algorithm to provide locations and sizes of the distributed storage systems; the second stage evaluates the fitness of the solution provided by the first part by solving a daily AC optimal power flow. An application example, referring to the IEEE 13 busses test feeder, is included in order to demonstrate and discuss the efficiency of the proposed method.

\section{INTRODUCTION}

Significant research and development has been conducted to improve cost, efficiency and reliability of energy storage systems in order to reach a certain technological maturity in relation to their use in electrical grids. However, a limited amount of literature has been produced in relation to the

The results described in this paper have been obtained within a research framework sponsored by the EOS Holding, Switzerland. 
optimal integration of Distributed Storage Systems (DSSs) in Active Distribution Networks (ADNs) (e.g., [1]). DSSs might have several potential uses ranging from network services (e.g. voltage control, management of line congestions, impact on network losses) to the local energy balance. In view of the above, the problem of DSSs sizing and siting into ADNs represents an important problem to be solved.

Currently, DSSs applications essentially refer to the problem of energy balance. Typical examples are: peakshaving (e.g., [2]) and renewables compensation (e.g. [3]). However, DSSs can also provide ancillary services like voltage and frequency control [4], [5] (this last is of particular interest in case of islanded networks).

On the contrary, the subject that refers to the optimal location and sizing of distributed generation (DG), has been largely discussed with reference to various goals (e.g., [6-8]). A methodology to allocate energy storage resources in order to decrease the wind energy curtailment and cost of energy supply has been presented in [9]. A hybrid genetic algorithm (GA) combined with a sequential quadratic programming algorithm has been proposed in [10] to size and site DGs, energy storages and reactive power compensation systems. The objective function proposed in [10] accounts: the network losses and the costs associated to the network upgrades and energy flow from the external grid.

In [11] the problem of the calculation of the total reserve provided by storage systems of a non-interconnected power network, with large penetration of renewables, is formulated. The peculiarity of the proposed approach consists in the use of the Discrete Fourier Transform (DFT) to determine the required balancing power in different time-spans. For each time-span (i.e., intra-day, intra-hour and real-time), the proposed approach identifies, by using the DFT-components, the total amount of power/energy required by energy storage systems.

Within this context, and following a preliminary study presented in [12] were a specific algorithm for the optimal location of DSSs to maximize their contribution to voltage control has been proposed, this paper extend such a methodology to account the inherent multi-objective advantages related to the usage of DSSs. In [12] voltage sensitivities coefficients, as a function of the nodal power injections, were used to determine the site of DSSs able to improve the network voltage profiles [13].

The extensions proposed in this paper are several. The first one refers to the determination of both optimal location and size of DSSs (whilst, in [12] the problem was limited to finding the optimal location only). The second one takes into account, together with the problem of the optimal voltage control, also the minimization of network losses and the energy cost from the external grid.

The problem is solved by using an iterative procedure based on two stages. In the first one the size and the location of DSSs are determined by using a heuristic approach based on the use of the Genetic Algorithm (GA). Once the GA solution is determined, a suitable fitness value of such a solution is calculated in a second stage by solving an $\mathrm{AC}$ optimal power flow (AC-OPF) problem. This second stage uses a suitably formulated multi-objective function that accounts the voltage deviations, the network losses and the cost of energy imported from the external grid.

The rest of the paper is organized as follows: Section 2 is devoted to the formulation of the problem. In Section 3, by making reference to a modified IEEE 13-bus test feeder, the result and efficiency of the methodology are presented. Section 4 concludes the paper with final remarks concerning the applicability of the proposed procedure.

\section{PRoblem Formulation}

The objective of the problem is to optimally site and size, into a given AND, a pre-determined amount DSS in terms of total installed power, energy and number of units. It is supposed that the ADN is connected to an external subtransmission grid characterized by a given hourly cost of the energy exchange. Additionally, as in [12], it is assumed that the various DSSs locations have equal installation and operation costs. Therefore, the objective function can be formulated as:

$$
\begin{gathered}
\text { Obj }=\left(W_{v o l}\left(\sum_{t} \sum_{i}\left(V_{i, t}-V_{\text {ref }}\right)^{2}\right)+W_{\text {loss }} \Gamma_{\text {loss }}\right. \\
\left.+W_{E P}\left(\sum_{t} C_{t}^{E} E_{t}\right)\right)
\end{gathered}
$$

The first term of (1) corresponds to the squared norm of voltage deviations of all the network nodes over a given period of time. The second term defines the total amount of the network losses over a given period of time. Finally, the third term corresponds to the total energy cost related to the electrical power flow with the external grid.

The weighting coefficients of each term of (1) have been determined by using the analytic hierarchy process (AHP) described in [7] and proposed by Thomas L. Satty [14].

The global constraints of the problem are:

- Maximum number of DSSs units installed in the ADN;

-DSSs operational and capacity constraints (i.e., energy reservoir bounds and DSS capability curves);

-Daily AC-optimal power flow (AC-OPF) constraints.

As it will be clarified next, the above-mentioned problem is a mixed-integer, non-convex and non-linear one. In order to deal with its inherent complex structure, a hybrid approach is proposed in this paper.

The scheme of the adopted approach is shown in Fig. 1. As it can be seen, a GA-based approach is used to provide a first solution of DSSs location and size. Subsequently, a subproblem is defined. It is associated to the building of a fitness function of the GA-solution determined by solving a daily AC-OPF. The GA algorithm and the daily AC-OPF are 
described in details in the coming sub-sections.



Figure 1. Schematic representation of the proposed DSS planning procedure.

\section{A. Genetic Algorithm approach for the solution of the master problem}

As above-mentioned, the master problem determines a first set of optimal locations and sizes of DSSs. A GA-based approach is used to solve this optimization problem and the fitness value is determined by the sum of installation costs and the objective value of a specific sub-problem described in the next sub-section. In view of what previously stated, the fitness function of the GA can be written as follows:

$$
F_{G A}=C_{I n v}+O b j_{s u b}
$$

Where, $C_{I n v}$ is the investment cost and $O b j_{s u b}$ is the objective value of the subproblem (i.e., (1)). The constraints are: (i) the total capacity of available DSSs, (ii) the total DSSs capacity that can be installed in each node, and (iii) the maximum number of nodes where DSSs can be installed.

\section{B. Fitness Function}

The fitness function uses the solution of the GA and performs a daily optimal AC load flow with a multi-objective function and then returns back the value of such an objective function to the GA (master problem).

The objective of the fitness function is as (1). The constraints are the load balance provided by the well-known power-flow equations and voltage upper/lower limits that, for the sake of clarity, are reported in (3)-(5) together with the DSSs constraints given in (6)-(10).

$$
\begin{aligned}
Q_{G_{i, t}}+Q_{D S S_{i, t}}-Q_{L_{i, t}} & =V_{i, t} \sum_{j}\left(V_{j, t}\left(G_{i, j} \sin \left(\delta_{i, t}-\delta_{j, t}\right)\right)\right. \\
& \left.\left.-B_{i, j} \cos \left(\delta_{i, t}-\delta_{j, t}\right)\right)\right) \\
P_{G_{i, t}}+P_{D S S_{i, t}}-P_{L_{i, t}} & =V_{i, t} \sum_{j}\left(V_{j, t}\left(G_{i, j} \cos \left(\delta_{i, t}-\delta_{j, t}\right)\right)\right. \\
& \left.\left.+B_{i, j} \sin \left(\delta_{i, t}-\delta_{j, t}\right)\right)\right)
\end{aligned}
$$

Voltage upper and lower limits:

$$
\begin{gathered}
V_{\text {min }} \leq V_{i, t} \leq V_{\max } \\
\sum_{t^{\prime}=0}^{t} P_{D S S_{i}}^{t^{\prime}} \Delta t \eta_{i} \leq E_{D S S_{i}}^{0} \quad \forall t, t=1,2, \ldots, 24
\end{gathered}
$$

$$
\begin{gathered}
\sum_{t^{\prime}=0}^{t}-P_{D S S_{i}}^{t^{\prime}} \Delta t \eta_{i} \leq E_{D S S_{i}}^{\max }-E_{D S S_{i}}^{0} \quad \forall t, t=1,2, \ldots, 24 \\
E_{D S S_{i}}^{\min } \leq E_{D S S_{i}}^{t} \leq E_{D S S_{i}}^{\max } \\
P_{D S S_{i}}^{\min } \leq P_{D S S_{i}}^{t} \leq P_{D S S_{i}}^{\max } \\
\left(P_{D S S_{i}}^{t}\right)^{2}+\left(Q_{D S S_{i}}^{t}\right)^{2} \leq\left(C_{D S S_{i}}^{\max }\right)^{2}
\end{gathered}
$$

Equations (6) and (7) refers to the maximum amount of energy that can be stored, or taken, from a generic DSS reservoir. It should be noted that we have assumed that the generation/absorption of reactive power from a DSS, does not affect its energy-reservoir level ${ }^{1}$. Equations (8) and (9) define the maximum and minimum capacity of the DSS reservoir as well as the relevant real-power rating. Equation (10) models the capability curve of a DSS based on the assumption that these devices are interfaced with the grid using a power electronic converter (see also footnote 1). In this respect, their capability curve is governed by the ampacity limit of the power converter that, in case of an operation under constant AC grid voltage, can be translated into a constraint on the apparent power delivered by the DSS (e.g., [15]).

The above-mentioned problem has been implemented within the MATLAB programming environment. The GA is solved with MATLAB GA optimization toolbox [16] and the daily AC-OPF problem is solved with MATLAB fmincon (find minimum of constrained nonlinear multivariable function) function [17].

\section{Simulation RESUltS}

The test system used to illustrate the proposed methodology is the IEEE 13 nodes test feeder which topology is depicted in Fig. 2 [18]. It is supposed to have nondispatchable DG units composed by photovoltaic panels (PVs).

Concerning the representation of the network loads, they are considered as voltage-independent $P Q$ absorbers. The time-series related to a daily total active/reactive load consumption and total PV production are shown in Fig 3. In particular, these profiles correspond to a typical summer and winter day. Concerning the reactive power injection of PVs, it is assumed null.

It is also assumed that the non-dispatchable PV injections are installed on nodes $3,6,8,11$, and 13 . The maximum number of nodes where DSSs could be installed is 3 , the assumed total DSSs power is equal to $600 \mathrm{~kW}$ with a relevant energy storage capacity of $3 \mathrm{MWh}$. Additionally, the overall efficiency of each DSS is considered to be $70 \%$ for both injecting and absorbing operation modes.

\footnotetext{
${ }^{1}$ It is worth observing that such an assumption is based on the fact that DSSs are interfaced with $\mathrm{AC}$ electrical grids towards power electronic converters. These devices are characterized, in general, by high efficiencies. Therefore, we assume, in a first approximation, that the generation/absorption of reactive power from DSSs does not influence their energy reservoir level.
} 


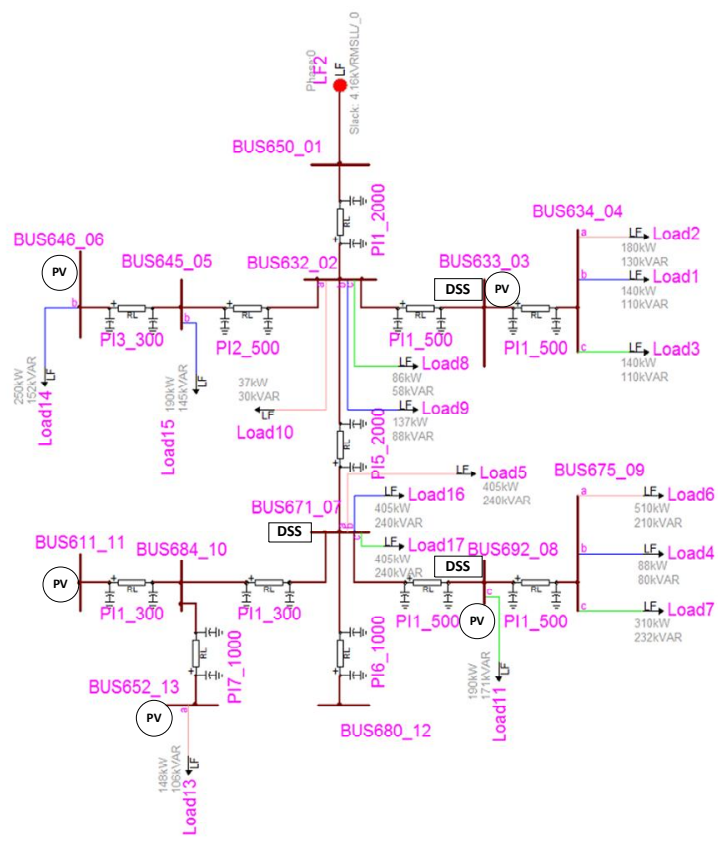

Figure 2. Topology of the IEEE 13 node test feeder [18].

TABLE I. OBTAINED OPTIMAL DSSS LOCATION AND SIZE.

\begin{tabular}{cccc}
\hline Node & 3 & 7 & 8 \\
\hline DSS power rating $\mathrm{kW}$ & 66 & 200 & 334 \\
\hline DSS reservoir capacity $\mathrm{kWh}$ & 670 & 1000 & 1330 \\
\hline
\end{tabular}

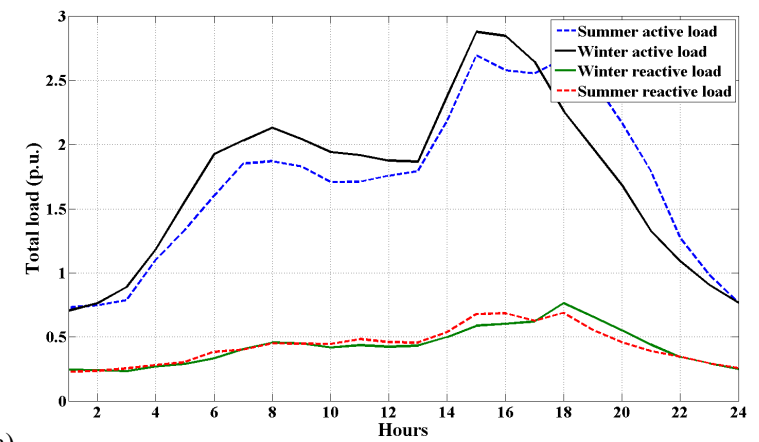

a)

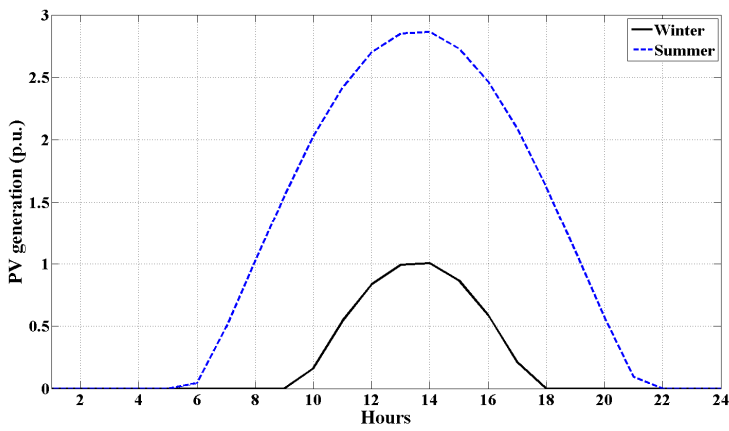

b)

Figure 3. Absorbed active and reactive power profiles of loads (a) and PV (b) for typical winter and summer days (base value of the power is 500 $\mathrm{kVA})$.

For each of both summer and winter scenarios a specific OPF problem can be formulated and solved. The objective values provided by these two scenarios are superposed and sent back to the GA stage towards the fitness function. The obtained optimal size and location of DSSs are shown in Table I. Concerning this aspect, it should be noted that the user can select any time horizon. In this respect we have decided to select two representative days for summer and winter.

In order to proof that the proposed process is able to satisfy the constraint concerning the DSS reservoir level (i.e., equations (6) and (7)), Fig. 4 shows this quantity concerning both summer and winter days. In particular, it is assumed that the energy reservoir level of each DSS, at the end of the considered time window, should be identical to the one at the beginning of the same time window (here assumed to be $40 \%$ of the maximum reservoir capacity). As it can be observed, the DSSs are charged during low load hours and discharged during peak load hours and return back to their original energy level. It is also interesting to observe that, due to the nature of the considered renewables, namely PVs, for the summer day the DSS are charged more compared to the winter day. Additionally, in the winter day the possibility of having DSSs reservoir levels close to zero is higher.
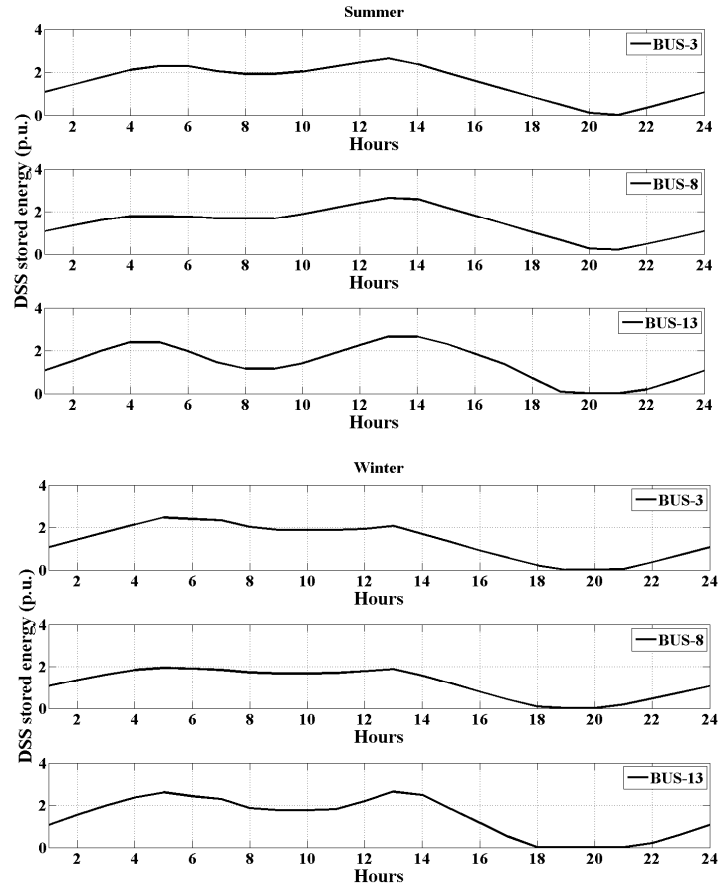

b)

Figure 4. Profiles of the energy reservoir levels of the various DSSs during summer (a) and winter (b) scenarios (base value of the is $500 \mathrm{kVA}$ ).

Fig. 5 reports the daily voltage profiles with and without the DSSs optimally planned with the proposed procedure. As it can be observed, the proposed procedure is capable of largely affect the quality of the voltage as, in both scenarios, the voltage deviations from the rated value do not exceed 3\%. On the contrary, the absence of the optimally planned DSSs results into voltage variations in the order of $10 \%$. 
In order to better analyze the performances of the proposed planning algorithm, Fig. 6 shows the probability density distributions of all the node voltages. As it can be seen, also these figures confirm the above-stated outcomes, namely: the presence of the optimally placed, and sized, DSSs allows to largely improve the quality of the voltage. In particular, the presence of DSSs allows obtaining voltage profiles that are always contained within the range 0.99 to 1.033 .

a)
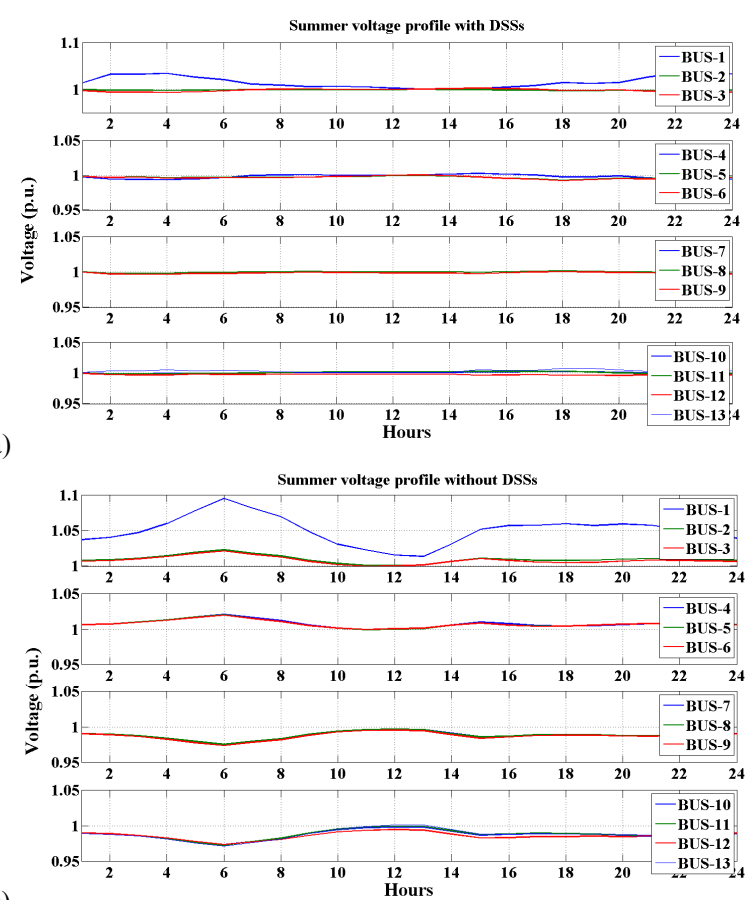

b)

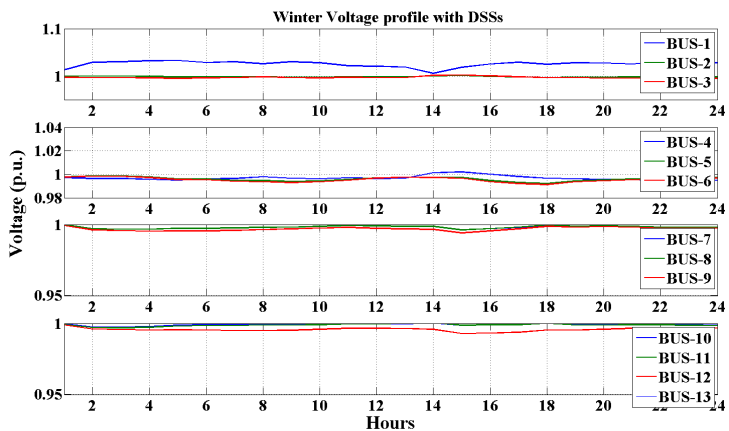

c)

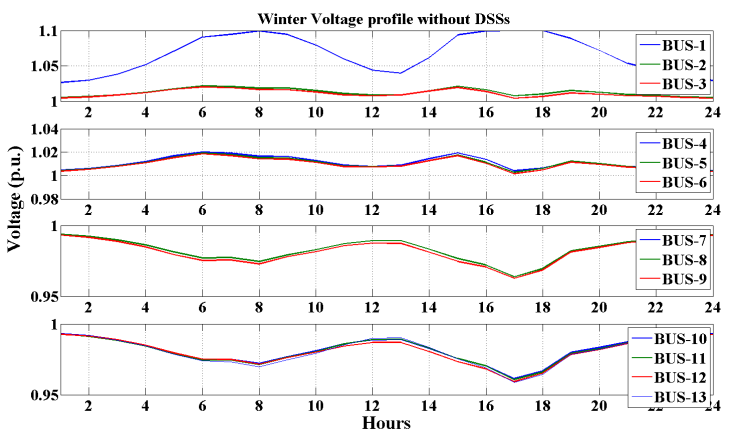

d)

Figure 5. Voltage profiles for all the network nodes with DSSs (a), (c), without DSSs (b), (d) for both summer (a), (b) and winter (c), (d) days.

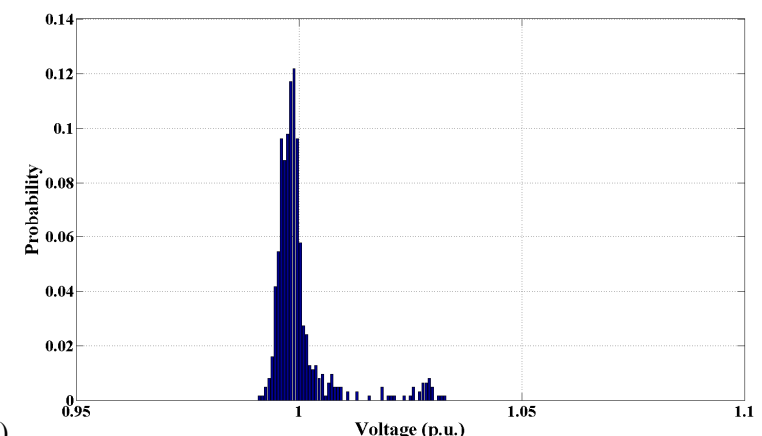

a)



Figure 6. Distribution of voltages in the network nodes within the considered time window: (a) with optimally placed DSS, (b) without any DSS.

The other two elements of the multi-objective function (1), namely the network losses and energy costs associated to the power flows with the external grid, are reported in Table II. As it can be seen, also these other two terms exhibit a large improvement for the case of optimally selected DSSs.

TABLE II. THE COST OF ENERGY AD LOSS IN BOTH CASES

\begin{tabular}{ccccc}
\hline Node & \multicolumn{2}{c}{ Loss (MWh/day) } & \multicolumn{2}{c}{$\begin{array}{c}\text { Cost of energy } \\
\text { (p.u. } \$ \text { /day) }\end{array}$} \\
\hline Period & winter & summer & winter & summer \\
\hline Without DSSs & 3.1 & 2.8 & 84.2 & 79 \\
\hline $\begin{array}{c}\text { Optimal selected } \\
\text { DSSs }\end{array}$ & 2.9 & 2.1 & 23.4 & 15.6 \\
\hline
\end{tabular}

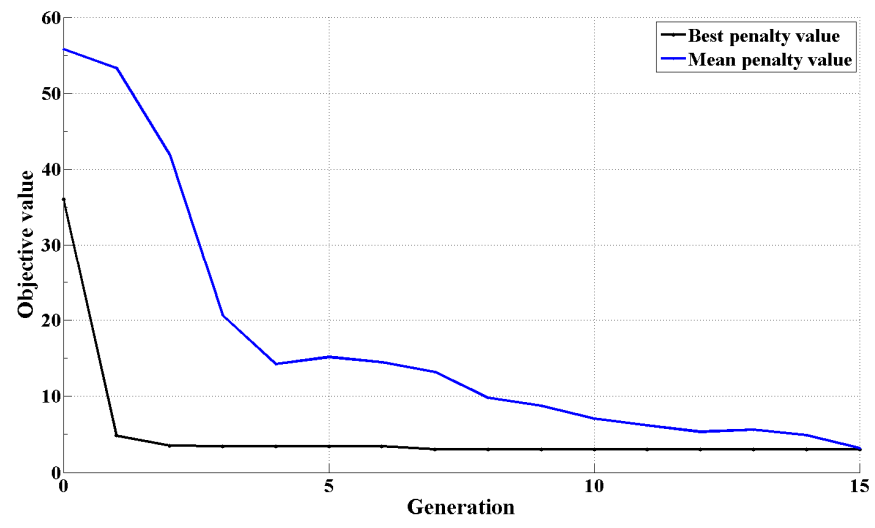

Figure 7. Trend of the mean and best values of the objective function of the daily $\mathrm{AC}-\mathrm{OPF}$ in each generation. 
The mean and the best penalty values of the GA that correspond to the amount of the objective function of the daily AC-OPF are shown in Fig. 7. It shows the convergence and the problem towards an optimal solution.

\section{CONCLUSION}

The paper has focused on the description of an optimal planning procedure applied to the location and sizing of DSSs with the purpose of maximizing their impact in terms of voltage support, loss minimization and energy cost from the grid to active distribution networks.

In view of the inherent complexity of the formulated problem, characterized indeed by a mixed-integer, non-linear and non-convex nature, a specific two-stage procedure has been proposed. It is composed by a two-stage iterative procedure where the first part utilizes a GA to provide locations and sizes of the DSSs whilst the second part evaluates the fitness of the solution provided by the first part by solving a daily AC-OPF.

In order to demonstrate the capability of the proposed procedure to find an optimal solution concerning the location and size of the DSSs, it has been applied to the IEEE 13 bus test feeder were load and non-dispatchable absorption and generation profiles were assumed for both summer and winter daily profiles. The application of the proposed procedure to the defined scenario has shown evident improvements of all the terms taken into account within the multi-objective function, namely the network voltages, the network losses as well as the cost of imported energy from the external grid.

In view of the above, we can conclude that the proposed procedure represents a consistent approach capable of accounting both DSSs contributions to the energy balance and, also, to their support to grids ancillary services.

\section{REFERENCES}

[1] M. Hoffmann , A. Sadovsky , M. Kintner-Meyer , J. DeSteese, "Analysis Tools for Sizing and Placement of Energy Storage for Grid Applications" U.S. Department of Energy, Springfield, Tech. Rep. PNNL-19703, Sep. 2010

[2] A. Oudalov, R. Cherkaoui, A. Beguin, "Sizing and optimal operation of battery energy storage system for peak shaving application", Prof. of the 2007 IEEE Lausanne Powertech, pp. $621-625$.

[3] J. P. Barton, and D. G. Infield, "Energy storage and its use with intermittent renewable energy," IEEE Trans. Energy Conv., vol. 19, pp. 441-448, Jun. 2004.

[4] M. Zillmann, Y. Ruifeng, T.K. Saha, "Regulation of distribution network voltage using dispersed battery storage systems: A case study of a rural network", Proc. of the 2011 IEEE PES General Meeting, pp. $1-8$.

[5] P. Mercier, R. Cherkaoui, A. Oudalov, "Optimizing a battery energy storage system for frequency control application in an isolated power system," IEEE Trans. Power Syst., vol. 24, pp. 1469 - 1477, Aug. 2009.

[6] N. Acharya, P. Mahat, N. Mithulananthan "An analytical approach for DG allocation in primary distribution network" Electrical Power and Energy Systems, vol. 28, pp. 669-678, Dec. 2006.

[7] Z. Liu, F. Wen, G. Ledwich, "Optimal siting and sizing of distributed generators in distribution systems considering uncertainties," IEEE Trans. Power Delivery, vol. 26, pp. 2541-2551, Oct. 2011.

[8] D. Gautam, N. Mithulananthan "Optimal DG placement in deregulated electricity market," Electric Power Systems Research, vol. 77, pp. 1627-1636, Oct. 2007.

[9] Y. Atwa, E. El-Saadany, "Optimal allocation of ESS in distribution systems with a high penetration of wind energy," IEEE Trans. Power Syst., vol. 25, pp. 1815-1822, November 2010.

[10] G. Carpinelli, F. Mottola, D. Proto, A. Russo "Optimal Allocation of Dispersed Generators, Capacitors and Distributed Energy Storage Systems in Distribution Networks" Modern Electric Power Systems 2010, pp. 1-6

[11] Y. Makarov, P. Du, M. Kintner-Meyer, C. Jin, H. Illian, "Sizing Energy Storage to Accommodate High Penetration of Variable Energy Resources" IEEE Trans. Sust. Energy, vol. 3, Jan. 2012

[12] M. Nick, M.Hohmman, R. Cherkaoui, and M. Paolone, "On the Optimal Placement of Distributed Storage Systems for Voltage Control in Active Distribution Networks", Proc. of the 2012 IEEE Int. Conf. on Smart Grids Technologies (ISGT) Europe, pp. 1-6.

[13] K. Christakou, J. Le Boudec, M. Paolone, and D. Tomozei, "Efficient Computation of Sensitivity Coefficients of Node Voltages and Line Currents in Unbalanced Radial Electrical Distribution Networks" Accepted for the publication in IEEE Trans. Smart Grid, 2013.

[14] Thomas L. Saaty 'Decision making - the Analytic Hierarchy and Network Processes (AHP/ANP) 'Journal of Systems Science and Systems Engineering, vol. 13, pp. 1-35, Mar. 2004,

[15] E. Muljadi, C.P. Butterfield, R. Yinger, and H. Romanowitz "Energy storage and reactive power compensator in a large wind farm" 42nd AIAA Aerospace Sciences Meeting and Exhibit Reno, pp. 1-9.

[16] "Genetic Algorithm Examples::Using the Genetic Algorithm," Mathworks Inc., 1984-2012. [Online]. Available: http://www.mathworks.ch/help/toolbox/gads/f6691.html.

[17] MathWorks, "Description of the Nonlinear Constraint Solver," MathWorks Inc., 1984-2012. [Online]. Available: http://www.mathworks.ch/help/toolbox/gads/bqf8bdd.html.

[18] IEEE Distribution Planning Working Group, "Radial distribution test feeders," IEEE Trans. Power Syst., vol. 6, pp. 975-985, Aug. 1991. 